Strauss, B. S. \& Pierog, S. (1954). J. gen. Microbiol. 10, 221-235.

\title{
Gene Interactions: the Mode of Action of the Suppressor of Acetate-requiring Mutants of Neurospora crassa
}

\author{
By B. S. STRAUSS AND SOPHIE PIEROG \\ Department of Zoology, Syracuse University, Syracuse 10, New York, U.S.A.
}

SUMMARY : Certain single-gene acetate-requiring mutants of Neurospora crassa (ac) are deficient in their ability to oxidize pyruvate directly, but can decarboxylate pyruvate to acetaldehyde and can form ethanol. The growth characteristics of the $a c$ mutants indicate that they can form acetate from glucose or ethanol. ac Mutants are inhibited by glucose or sucrose. This glucose inhibition is relieved by the singlegene suppressor mutations $s p$ and $c a r$ which lower the activity of pyruvic carboxylase, decrease the amount of ethanol formed and permit some growth of $a c s p$ and ac car strains in absence of added acetate. The $s p$ mutation also lowers ethanol dehydrogenase activity, but this activity can be partially restored by growth of $s p$ strains in the presence of ethanol. As a result of the nearly complete block in their pyruvate metabolism, ac sp strains are forced to metabolize glucose by pathways which produce more of the initial respiratory $\mathrm{CO}_{2}$ from the C-1 carbon of glucose.

While genetic theory is reticent about the embodiment of the gene (Pontecorvo, 1952) there is little doubt that the physiological effects of genes are mediated via enzymes. Genes, individually and en masse, control the rates of the step reactions from which multi-enzyme systems (Dixon, 1949) are built up, and in so doing determine the metabolic pathways chosen by the organism. In many instances gene mutation causes the block, complete or incomplete, of a step reaction; the consequence of such a block is the accumulation of precursors and the appearance of a growth requirement for the product of the blocked reaction (Beadle, 1945).

The commonest use for genetic mutants in biochemistry is the definition of routes of biosynthesis by a study of the growth requirements induced and the identification of the precursors accumulated (Horowitz, 1950). As interesting, perhaps, are the inhibitions produced by the accumulated precursors or by 'abnormal' products derived from these precursors (Emerson, 1949; Bonner, 1946). If genes do control the rates of step reactions, it should be possible with a second mutation to relieve an inhibition induced by an initial mutation, if the second mutation prevents the formation of the inhibitor. Such relief by a second mutation would be denoted, genetically, as a suppressor or modifier effect and would be one of the many instances in which the effects of two co-existing mutant genes are not merely additive. The gene interaction in such a situation would not be the simple interaction of two genes present in the nucleus but would, rather, express the interaction of the enzymic reaction systems present in non-genic parts of the cell; such interactions would further demonstrate the interdependence of the biochemical reactions of the organism. These investigations demonstrate an example of gene interaction in wellknown metabolic pathways and indicate that the selection of one of several alternate pathways is here under genetic control; all this would again emphasize the importance to genetical theory of inhibition by naturally-occurring 
intermediates. Single-gene mutants of the Ascomycete Neurospora crassa which require acetate (Lein, Appleby \& Lein, 1951; Lein \& Lein, 1952; Strauss, 1952, $1953 a, b$ ) were used in the present work; they were selected because of the wealth of knowledge of alternate pathways of carbohydrate metabolism and because of the obvious importance of carbohydrate metabolism in the economy of $N$. crassa. These acetate-requiring mutants are inhibited by glucose. A second type of single-gene mutant with no acetate requirement was used; this latter kind of mutant is characterized by a low pyruvic carboxylase activity and lowered ethanol production as compared to the wild type. When these two mutants are genetically combined, the double mutants obtained grow slightly on media not containing acetate ('suppressor' action) and are no longer inhibited by glucose.

That the suppressor gene acts by lowering the rate of formation of an inhibitor formed as a result of the primary mutation to acetate-dependence is the conclusion drawn from these investigations.

\section{METHODS}

The technique for growing neurospora was described previously (Beadle \& Tatum, 1945). Volatile or heat-labile compounds were sterilized by filtration through a Seitz filter.

Acetic acid was determined by titration of an 11 vol. steam distillate; the identity of the volatile acid was checked by Duclaux distillation when necessary. 3-Hydroxy-2-butanone [acetylmethylcarbinol (AMC)] was determined by the method of Westerfeld (1945); acetaldehyde by the method of Barker \& Summerson (1941); ethanol by oxidation of volatile neutral substances with dichromate and sulphuric acid, except when it was desired to eliminate AMC in which case a preliminary distillation from alkaline mercuric salts was performed as described by Friedemann \& Klass (1936). Glucose was determined by the Shaffer-Somogyi method (1933); phosphorus by the method of Fiske \& SubbaRow (1925); protein $\mathbf{N}$ by digestion of a trichloracetic precipitate (Ballentine \& Gregg, 1947); pyruvate by the method of Friedemann \& Haugen (1943).

Enzyme assays were made on the supernatant from a homogenate made by suspending mycelium in three times its wet weight of cold buffer, grinding in a glass homogenizer, and then centrifuging at $0^{\circ}$ for $10 \mathrm{~min}$. at $3750 \mathrm{~g}$. Ethanol dehydrogenase was assayed as described by Nason, Kaplan \& Colowick (1951) at $\mathrm{pH} \mathrm{7.6}$ [tris-(hydroxymethyl)-aminomethane buffer, Sigma Chemical Co.]. Aldolase was assayed at pH 8.6 in 'tris' buffer by the method of Sibley \& Lehninger (1949). This method was checked for N. crassa by studying the accumulation of alkali-labile phosphorus after incubation with hexose diphosphate as described below. Glucose-6-phosphate dehydrogenase was assayed by measurement of the increase in absorption of triphosphopyridinenucleotide (TPN) at $340 \mathrm{~m} \mu$. upon incubation with enzyme and 0.0045 M-glucose-6-phosphate substrate in $\mathrm{pH} \mathrm{7.0}$ phosphate buffer (H. K. Mitchell, personal communication).

${ }^{14}$ Carbon assays were made with a Radiation Counter Laboratories flow 
counter operating in the Geiger region with a gas mixture of helium +isobutane in conjunction with a Tracerlab 'Utility' scaler. Self-absorption corrections were made by using an empirical curve of absorption by $\mathrm{CH}_{3}{ }^{14} \mathrm{COOH}$. Counts were made at at least 10 times the background rate, and sufficient counts were recorded to insure a probable error due to the counting of less than $1 \%$. The ${ }^{1-14} \mathrm{C}$-labelled glucose was obtained from the National Bureau of Standards.

\section{MATERIALS}

Organisms. The acetate-requiring mutants of $N$. crassa used in these studies were described previously under the strain designations UT 50-4, S 34, S 48, $\mathrm{S} \mathrm{53,}$, and S210. For convenience these will be referred to as $a c-1,-2,-3,-4,-5$ respectively. The low-carboxylase strain $\operatorname{car}$ (Strauss, 1952) and the suppressor strain $s p$ (Lein \& Lein, 1952) will be referred to by these designations. Strains $a c-2,-3,-5$ have been shown to be non-allelic, while strains $a c-1$ and $a c-4$ can be distinguished phenotypically from the others and from each other. These latter strains will not grow on glucose + acetate as do the others but do grow on $\mathrm{D}(+)$ xylose + acetate. None of the ac mutants responds to synthetic 6,8-thioctic acid nor to yeast extract in catalytic concentrations.

Table 1. Results of crossing ac, sp and car strains

$\begin{array}{cc}\begin{array}{c}\text { Genotypes of } \\ \text { parental strains } \\ a c-4 s p+\end{array} & \begin{array}{c}\text { Genotypes of } \\ \text { segregants } \\ a c+s p \\ a c+s p+ \\ a c-4 s p\end{array} \\ \times & \begin{array}{c}a c-4 s p+ \\ a c+s p\end{array} \\ a c+c a r+s p+ & \left.\begin{array}{l}a c+c a r+s p+ \\ a c+c a r s p \\ a c+c a r s p+ \\ a c+c a r+s p \\ a c-1 \text { car } s p \\ a c-1 \text { car }+s p \\ a c-1 \text { car } s p+\end{array}\right\}\end{array}$

$a c-1$ car $s p+\quad a c-1 c a r+s p+$

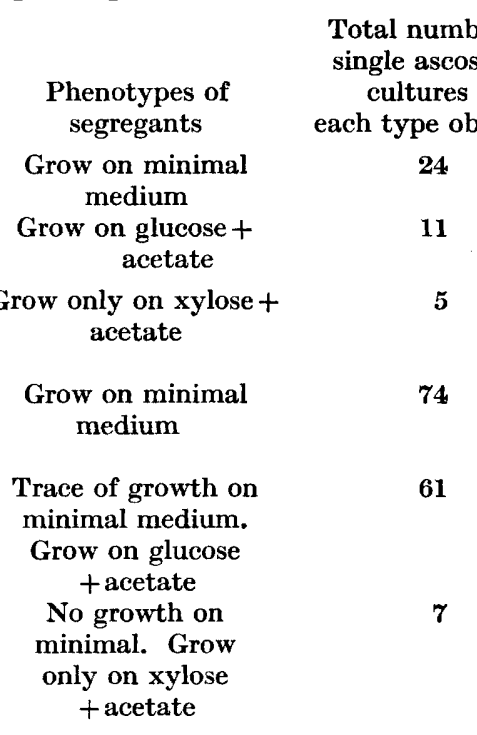

Total number of le ascospore ype obtained

\section{4} 11 5 7

$F_{1}$ data were obtained by classifying cultures obtained from single ascospores derived from the appropriate cross. The total sample of viable ascospores is the sum of the segregants in each case.

The $s p$ strain was described (Lein \& Lein, 1952) as permitting some growth on minimal medium when combined with $a c-2,-3$, or -5 . Later (Strauss, 1953 $a$ ) it was shown that this $s p$ strain carried with it low carboxylase activity with concomitant low ethanol production. It can also be demonstrated that the $s p$ gene relieves the extreme glucose inhibition characteristic of $a c-4$ so that $a c-4 s p$ can grow on glucose + acetate (Table 1). The $s p$ strain is therefore pheno- 
typically quite similar in its action to the car strain, since both have low carboxylase activity and both relieve the extreme glucose inhibitions of strains $a c-1$ and $a c-4$. Nevertheless, these two genes are not alleles (Table 1). The cross $a c-1 c a r \times a c+s p$ leads to the formation of strains that do not grow on glucose + acetate but which will grow on xylose + acetate or on acetate alone. The simplest explanation is that $s p$ and $c a r$ are non-allelic.

Table 2. Accumulation of pyruvic acid, ethanol and 3-hydroxy2-butanone by resting cultures

Cultures incubated $16 \mathrm{hr}$. in $15 \mathrm{ml}$. pH 6.0 $\mathrm{PO}_{4}$ buffer $+1 \%$ glucose after growth for 4 days and dissimilation in buffer for $24 \mathrm{hr}$.

Compound accumulated

Pyruvic acid

3-Hydroxy-2-butanone

Ethanol

\begin{tabular}{|c|c|}
\hline \multicolumn{2}{|c|}{ Strain } \\
\hline $\begin{array}{l}a c-5 s p+ \\
\mu \mathrm{M} / \mathrm{mg} . \mathrm{dry}\end{array}$ & $\begin{array}{r}a c-5 s p \\
\text { wt. cells }\end{array}$ \\
\hline $1.6 \times 10^{-2}$ & $7 \cdot 4 \times 10^{-2}$ \\
\hline $1.9 \times 10^{-2}$ & $5.7 \times 10^{-2}$ \\
\hline $7 \cdot 6$ & $2 \cdot 6$ \\
\hline
\end{tabular}

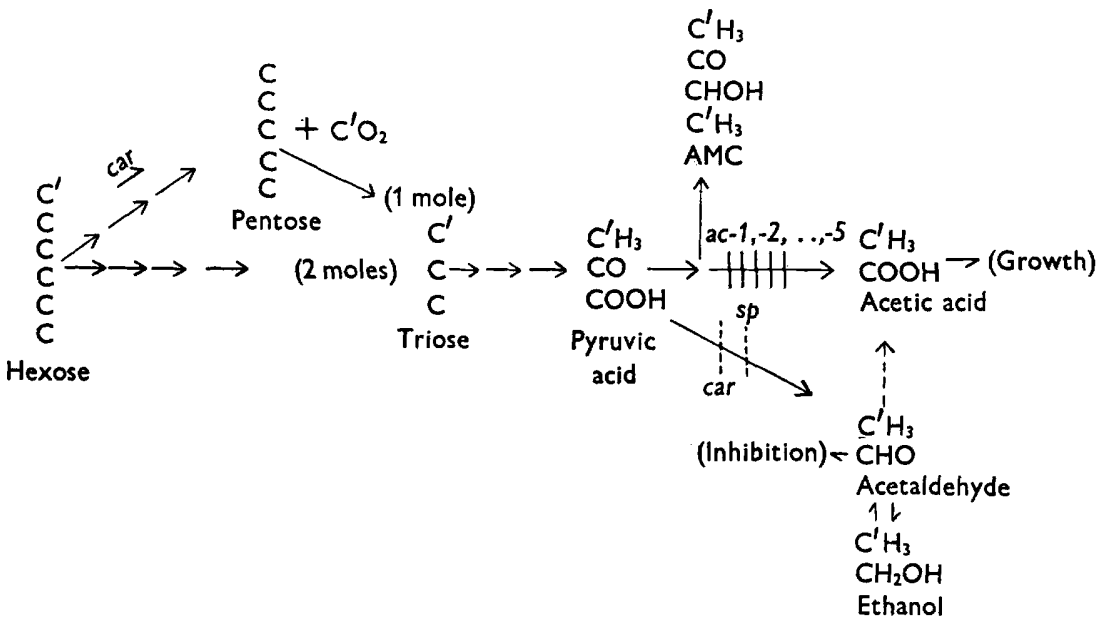

Fig. 1. Pathways of carbohydrate metabolism of importance to the ac mutants of Neurospora crassa

The strains used have the following biochemical characteristics: $a c$ strains require acetate; some growth of $a c s p$ or $a c$ car strains does occur on minimal medium but even these strains respond to added acetate by a relatively large increase in growth rate; all $a c s p+$ and $a c c a r+$ strains are inhibited to some degree by glucose (see below); ac strains are deficient in their ability to oxidize pyruvic acid (Strauss, 1953 $b$ ) and accumulate pyruvic acid and AMC (Table 2). $c a r$ and $s p$ strains have low pyruvic carboxylase activity and $a c s p$ strains accumulate larger quantities of pyruvate and AMC than do ac sp + strains which are ethanol accumulators. These properties are summarized in Fig. 1. 


\section{RESULTS}

\section{Glucose inhibition}

Strains $a c-1$ and $a c-4$ will not grow in the presence of glucose or sucrose but will grow on $\mathrm{D}(+)$ xylose + acetate. Both $s p$ and car relieve this inhibition, and it has been suggested (Strauss, 1953b) that the partial block in the pyruvic carboxylase reaction, produced nutritionally by growth on xylose, or genetically by the introduction of $c a r$ or $s p$, is biochemically involved in the relief from glucose inhibition. Strains $a c-2,-3$ and $a c-4$ grow on glucose, yet they, too, are inhibited by glucose and sucrose (Table 3 ). This inhibition is overcome as the culture ages and is similarly overcome by the introduction of $s p$ (Fig. 2).

\section{Table 3. Inhibitory effect of glucose and sucrose on acetate- requiring mutants}

Medium : $20 \mathrm{mI}$. Fries $+20 \mathrm{mg}$. acetate. Yield of growth recorded mg. dry wt. after 3 days growth at $25^{\circ}$. Note: $a c-1$ and $a c-4$ do not grow at all on sucrose or glucose but do grow with xylose + acetate.

\begin{tabular}{|c|c|c|c|c|}
\hline \multirow{3}{*}{ Sugar } & \multicolumn{4}{|c|}{ Strain } \\
\hline & $a c-2 s p+$ & $a c-3 s p+$ & $a c-5 s p+$ & $a c-5 s p$ \\
\hline & \multicolumn{4}{|c|}{ Yield of organism (mg. dry wt.) } \\
\hline Glucose, $1 \%$ & $12 \cdot 4$ & $0 \cdot 8$ & 0 & $28 \cdot 1$ \\
\hline Sucrose, $1 \%$ & $25 \cdot 7$ & $\mathbf{2 4} \cdot \mathbf{2}$ & $4 \cdot 0$ & $27 \cdot 0$ \\
\hline Sucrose, $3 \%$ & $16 \cdot 2$ & $4 \cdot 7$ & $3 \cdot 2$ & $27 \cdot 2$ \\
\hline Xylose, $1 \%$ & $33 \cdot 2$ & $38 \cdot 1$ & $23 \cdot 4$ & $27 \cdot 8$ \\
\hline
\end{tabular}

An attempt was made to trace the site of glucose inhibition by testing the inhibitory effects of substances related to the carboxylase reaction (Fig. 3). Pyruvate is inhibitory to $a c-3 s p+$ grown in media containing $2 \%$ sucrose but is stimulatory when tested in $1 \%$ xylose media. Strain $a c-3 s p$ is not significantly affected by pyruvate in either xylose or sucrose media. Ethanol is more inhibitory to $a c-3 s p+$ on sucrose media than on xylose, and low ethanol concentrations stimulate $a c-3 s p+$ slightly in xylose media. Strain $a c-3 s p$ is greatly stimulated by ethanol, and the response of this strain to ethanol is identical on both xylose and sucrose media.

The response of $s p$ and $s p+$ strains to pyruvate and to ethanol suggests that the effect of these compounds is related and additive to the inhibitory effect of sucrose or glucose. Only the strains inhibited by sucrose are inhibited by pyruvate or by ethanol, and these compounds are not as inhibitory to $s p+$ strains on xylose as on sucrose media. These considerations point to acetaldehyde (or some closely related substance) as the basis of 'glucose inhibition', since acetaldehyde is formed from pyruvate via the carboxylase reaction and from ethanol by ethanol dehydrogenase.

Acetaldehyde is inhibitory to $a c-1 c a r+$ (Fig. 4) and to the $s p+$ strains, although more acetaldehyde is required for inhibition of these strains. Because of the volatility of acetaldehyde, the concentration values given are only 


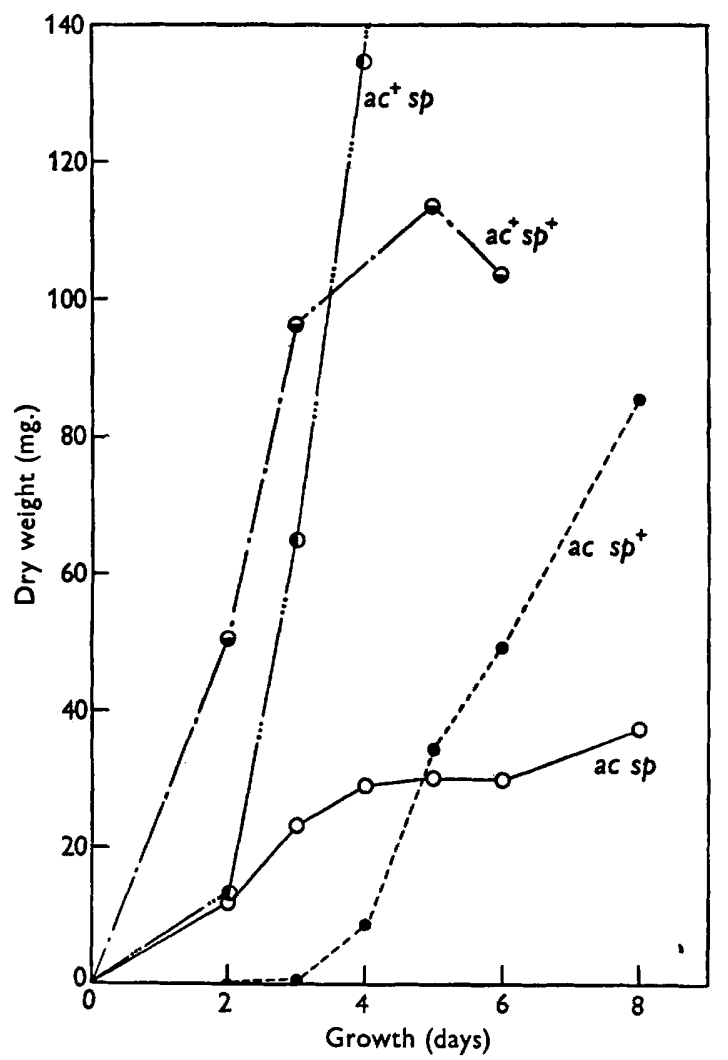

Fig. 2. Growth of $a c$ mutants as a function of time. Medium: $20 \mathrm{ml}$. minimal medium $(2 \%$ sucrose $)+20 \mathrm{mg}$. acetate. $a c-3 s p$ and $a c-3 s p$ used; similar results are obtained with $a c-2$ and $a c-5$.

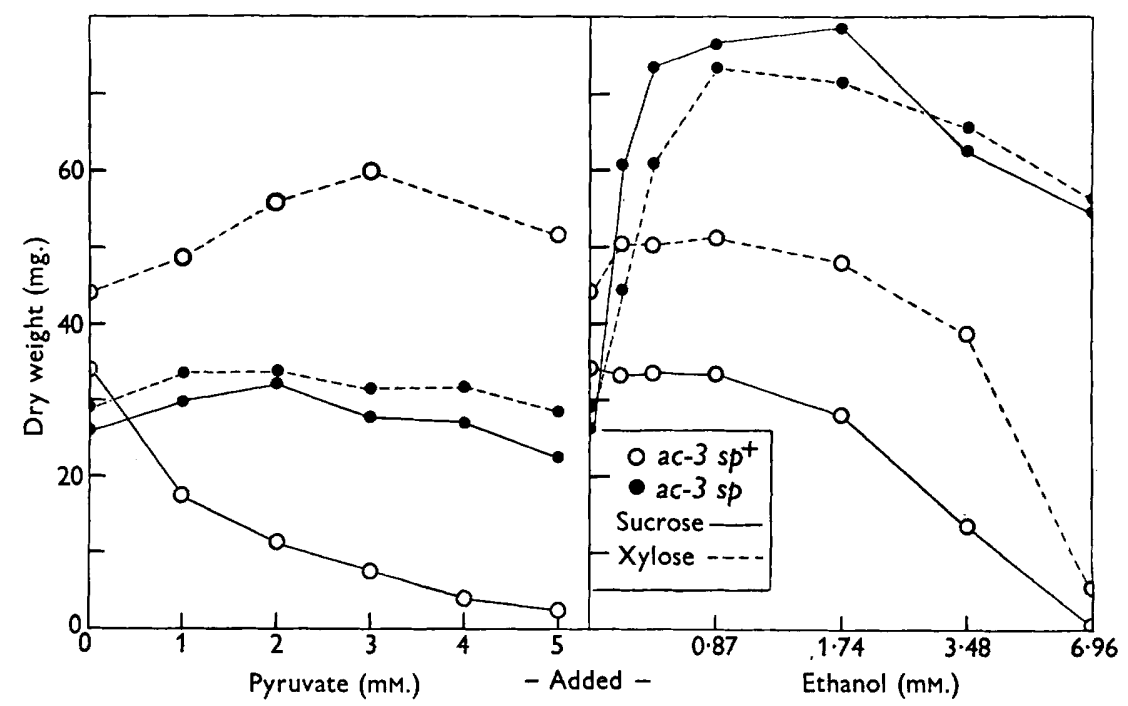

Fig. 3. Effect of added pyruvate and ethanol on $a c$ strains. Medium : $20 \mathrm{ml}$. Fries $+20 \mathrm{mg}$. acetate $+1 \% \mathrm{D}(+)$-xylose or $2 \%$ sucrose as indicated. Growth period: $72 \mathrm{hr}$. at $25^{\circ}$. 
approximate. After standing 2 days at $25^{\circ}$ a non-inoculated medium to which acetaldehyde had been added contained almost no detectable acetaldehyde. Paraldehyde is not inhibitory to $N$. crassa at these concentrations.

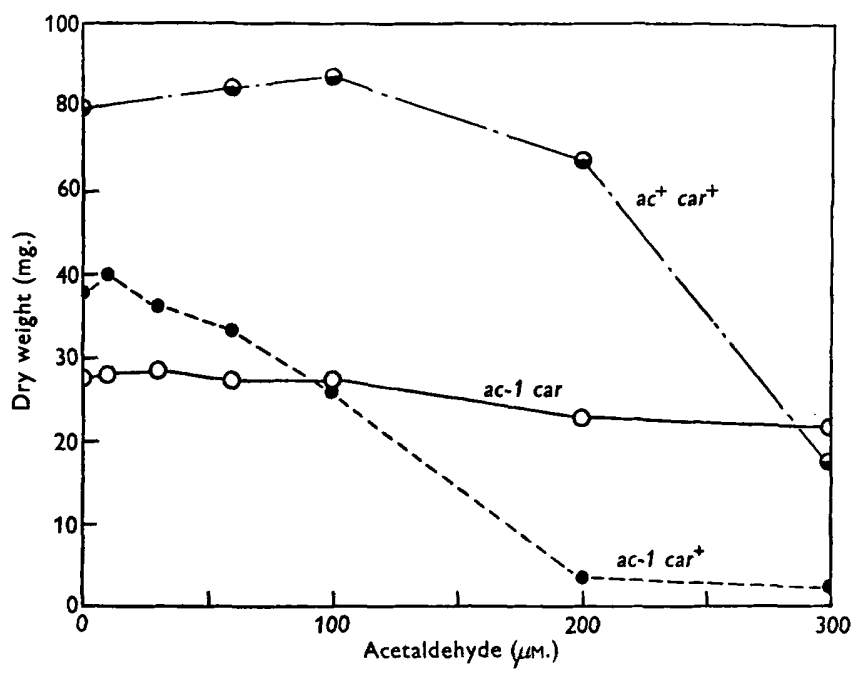

Fig. 4. Effect of added acetaldehyde on ac strains. Medium: $20 \mathrm{ml}$. Fries $+20 \mathrm{mg}$. acetate $+1 \%$ xylose. Growth period: $72 \mathrm{hr}$. at $25^{\circ}$.

\section{Acetate production}

Lein \& Lein (1952) showed that although $a c s p$ strains will grow slightly on minimal medium, in a medium containing acetate $a c s p$ strains grow less in a 5-day growth period than the corresponding ac $s p+$ strains. This behaviour was investigated by studying the growth of $a c-3 s p$ and $a c-3 s p+$ strains as a function of time in media containing a limiting amount of acetate (Fig. 2). Similar growth curves are obtained with $a c-2,-3$ and $a c-5$.

Although $a c s p$ strains show growth more rapidly than $s p+$ strains, growth slows after exhaustion of the added acetate from the medium at 3-4 days. The lag which $a c s p+$ strains show before growth is initiated is probably due to inhibition by sugar in the medium. Once this inhibition is overcome, ac sp+ grows at a rate approaching that of the wild type. No appreciable volatile acid accumulates in the medium during the rapid period of growth after the $a c s p+$ strain has overtaken the $a c s p$ strain. The rapidly growing $a c s p+$ is still an acetate-requiring mutant since subcultures of the rapidly growing mycelium made on acetate medium and then transferred to minimal medium do not grow unless acetate is added. Decreasing the amount of acetate added to $a c s p+$ strains has no effect on the total crop of mycelium eventually obtained. Cultures of $a c-3 s p+$ fed one-fortieth the amount of acetate which produces maximum growth in a 4-day period eventually attained the wild-type yield of mycelium, although over 2 weeks were required. The mycelium obtained in this last case was shown to be that of an acetate-requiring mutant. $a c s p+$ Strains accumulate considerable amounts of ethanol as compared to 
their ac $s p$ counterparts, and the rapid increase in dry weight of $s p+$ strains is accompanied by the appearance of ethanol in the medium (Fig. 5). The addition of ethanol to $a c s p$ cultures after 2 days growth causes them to duplicate the rapid growth characteristic of $a c s p+$. In the absence of other carbon sources all $a c$ strains give a few milligrams of growth with ethanol as well as with acetate. In the presence of sugar, ethanol can be used as a substitute for acetate by $a c s p$ strains (Fig. 6), but no growth of $a c s p+$ on

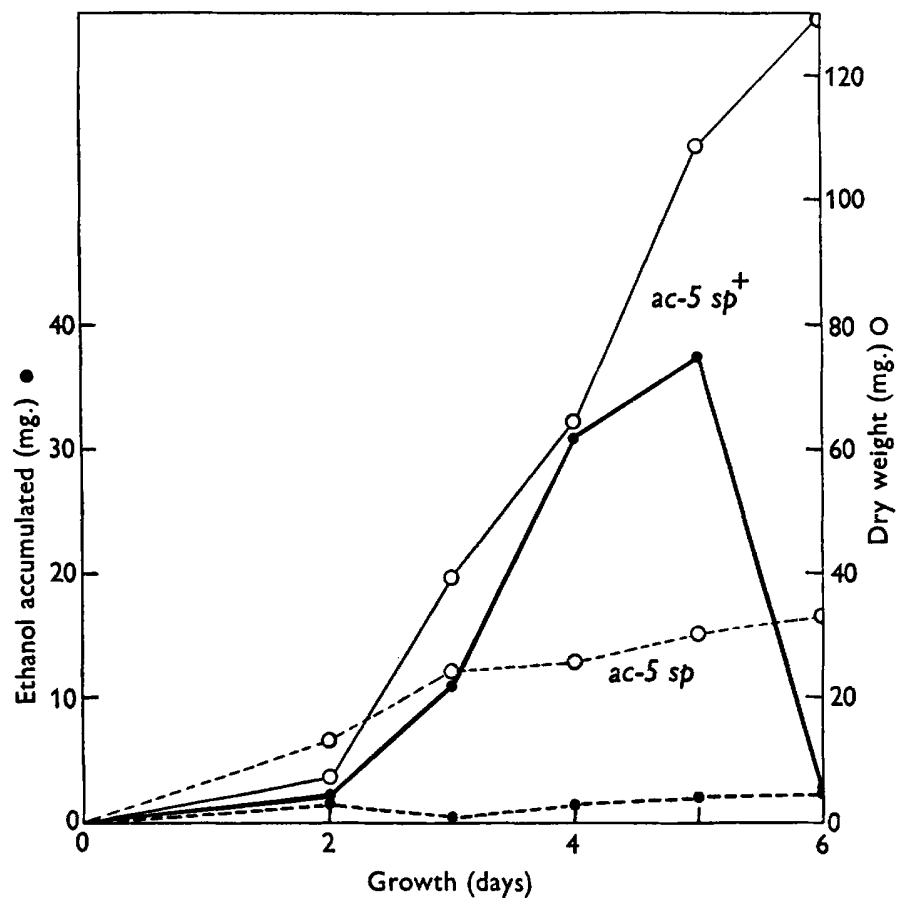

Fig. 5. Ethanol accumulation and growth as a function of time for ac strains. Medium: $20 \mathrm{ml}$. minimal (2\% sucrose) $+20 \mathrm{mg}$. acetate.

ethanol in the absence of acetate has ever been obtained. A previous report (Strauss, 1952) that ethanol could not be used for growth initiation by ac-1 car was due to the use of a standard 3-day growth period. There is a lag before growth begins on ethanol, and this seems to be related to the time required for the appearance of a visible amount of growth from a control inoculum on minimal medium. It is also evident from the data that the effect of ethanol and acetate together is more than additive.

These data indicate that acetate (or its active form) can be synthesized from glucose or from ethanol by ac mutants. Further evidence for this hypothesis is given in Table 4. Glucose lowers the amount of acetate taken up by $a c s p+$ strains but not by $a c s p$ strains, which require the addition of ethanol in order to decrease acetate uptake. This is not a case of glucose inhibition, since it can be shown that $a c s p+$ strains take up a greater amount of glucose than do ac sp. 
An attempt was made to demonstrate the formation of acetate by utilizing the methods of isotope dilution. No significant dilution of added $\mathrm{CH}_{3}{ }^{14} \mathrm{COOH}$ could be demonstrated when cultures of $a c s p+$ were grown for 4 days in labelled acetate + glucose, nor could any acetate of constant specific activity be

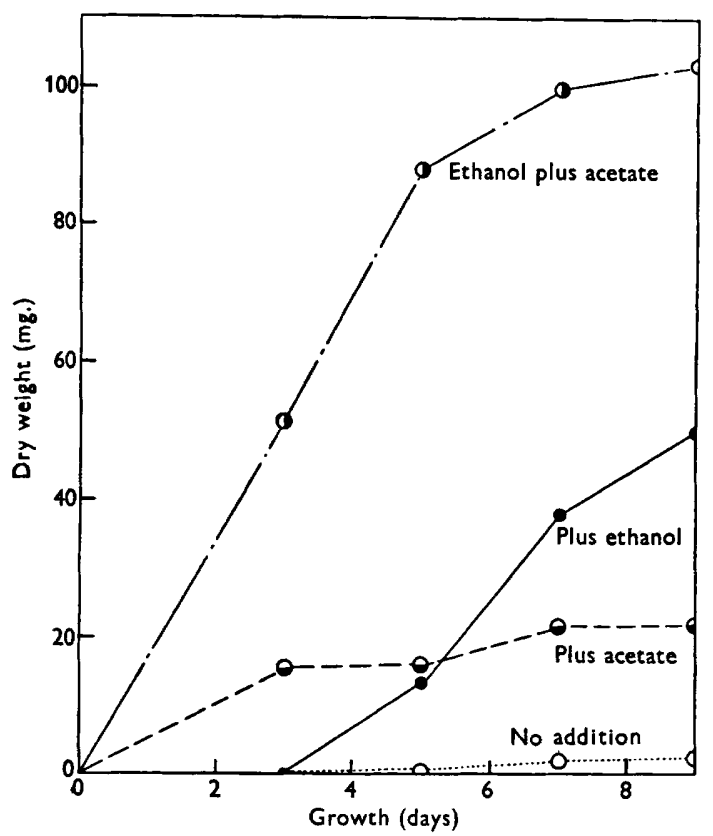

Fig. 6. Effect of ethanol on growth of ac-1 car. Medium: $20 \mathrm{ml}$. minimal (2\% sucrose). Ethanol, $40 \mathrm{mg}$. added. Acetate, $10 \mathrm{mg}$. added.

Table 4. Uptake of acetate by ac mutants

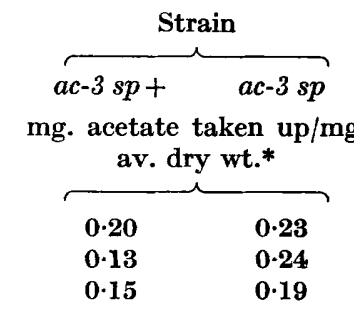

Suspending medium

Buffer $+100 \mathrm{mg}$. acetate

Buffer $+100 \mathrm{mg}$. acetate $+2 \mathrm{~g}$. glucose

Buffer $+100 \mathrm{mg}$. acetate $+2 \mathrm{~g}$. glucose + ng. acetate taken up/mg. $250 \mathrm{mg}$. ethanol

* Average dry wt. $=$ wt. at 0 time + wt. after $22 \mathrm{hr}$. incubation $/ 2$.

isolated from cultures fed acetate + glucose-1-14 $\mathrm{C}$. Wild-type cultures also failed to give evidence for acetate synthesis under these conditions. These experiments are therefore not considered evidence against the hypothesis of acetate formation by acetate-requiring mutants. 


\section{Alterations in metabolic pattern}

Previous work (Strauss, 1953 $b$ ) indicated that the lowering of pyruvic carboxylase activity by either genetic means (introduction of car) or nutritional means (growth on xylose) relieved the inhibition of $a c$ strains. $c a r+$ Strains have 4 to 6 times the carboxylase activity of car strains after growth on sucrose, but have at most twice the activity of car strains after growth on xylose. car Strains have the same carboxylase activity after growth on either sucrose or xylose. It was therefore suspected that the car gene (and analogously the $s p$ gene) might act by shunting metabolism to pentose. This would lead to the same effects on enzyme pattern as growth on xylose: lowered carboxylase formation with consequent relief from glucose inhibition. This idea was tested using glucose ${ }^{1-14} \mathrm{C}$. If $s p$ and $c a r$ strains metabolize glucose via a pentose shunt, then one would expect a relatively higher respiratory $\mathrm{CO}_{2}$ and a lower molar radioactivity in the ethanol accumulated by car strains than in that formed by car + strains, since in the operation of the EmbdenMeyerhof scheme the $C_{1}$ of glucose is retained in the methyl of pyruvate, AMC and ethanol (Fig. 1). The exact values obtained would be complicated by the factors of assimilation and $\mathrm{CO}_{2}$ fixation. These probably occur in the 18-20 hr. incubation period under aerobic conditions which is necessary to accumulate sufficient quantities of ethanol. For the comparison of different strains the ratio of ethanol activity to respiratory $\mathrm{CO}_{2}$ activity has been used as the most obvious method.

Mycelial pads were obtained after 4 days growth at $25^{\circ}$ in $20 \mathrm{ml}$. minimal medium containing $20 \mathrm{mg}$. acetic acid. The pads were then transferred to $20 \mathrm{ml}$. pH 6.0 M/15 phosphate buffer and allowed to dissimilate for $24 \mathrm{hr}$. Two or three pads were then transferred to $125 \mathrm{ml}$. flasks containing $20 \mathrm{ml}$. $1.5 \%$ glucose $-{ }^{14} \mathrm{C}_{1}$ in $\mathrm{M} / 30$ phosphate buffer. An inverted $U$ tube containing soda lime was attached to the flasks and a cup containing $4 \mathrm{ml}$. $2 \mathrm{~N}$-sodium hydroxide was suspended above the mycelium. The cultures were not shaken and it was not supposed that this method diminished the $\mathrm{CO}_{2}$ tension of the medium to zero. After 18-20 hr. incubation the absorbed $\mathrm{CO}_{2}$ was precipitated as $\mathrm{BaCO}_{3}$. The medium was neutralized, steam distilled, and the steam distillate was redistilled from a mixture of alkaline mercuric salts. A sample of this distillate was oxidized with acid dichromate and steam distilled. The distillate was neutralized with $\mathrm{NaOH}$, plated, and counted as sodium acetate. The specific activity of the glucose used was determined from its osazone. In all cases some glucose remained in the medium at the conclusion of the experiment. ac car and ac sp Strains gave higher respiratory $\mathrm{CO}_{2}$ and lower ethanol activities than did their $a c+$ counterparts (Table 5). This does not seem to be an effect of the $s p$ gene acting alone since $a c+s p$ gives activities closer to the wild-type and to $a c s p+$ values than to $a c s p$. There can be little doubt that ac sp strains metabolize glucose over quantitatively different pathways from those used by the wild-type, but this seems due to neither ac nor $s p$ gene alone but rather to their combination. The car gene behaves more nearly as predicted, since $a c+c a r$ gives a much lower ratio than does the wild-type and 
approaches the ratio given by ac car. In all cases studied the double mutants ac $s p$ or ac car give lower ratios than does any single mutants.

If the Embden-Meyerhof pathway were solely responsible for ethanol formation by any of the strains tested, the isolated ethanol should have an activity of $25 \times 10^{4}$ counts $/ \mathrm{min}$. $/ \mathrm{mm}$. Dilution of this by ethanol formed via carbon dioxide fixation or via the pentose shunt would lower the value obtained. It is possible that the low values obtained are the result of such dilutions. The exact values obtained may depend on the particular genetic background of the strain employed, since it is possible that all the factors leading to dilution of the labelled ethanol are genetically controlled.

\section{Table 5. Genetic alteration in the pattern of glucose metabolism}

Recorded counts $/ \mathrm{min} . / \mathrm{mm} \times 10^{-4}$. Glucose activity corrected to $5 \times 10^{5} \mathrm{counts} / \mathrm{min}$. $/ \mathrm{mm}$. Values are averages of duplicate experiments run simultaneously. $a c-1 c a r+$ and $a c-1 c a r$ grown on $1 \%$ xylose + acetate.

\begin{tabular}{|c|c|c|c|c|c|c|c|}
\hline & \multicolumn{7}{|c|}{ Strain } \\
\hline & $a c+c a r+s p+$ & $a c+s p$ & $a c-3 s p+$ & $a c-3 s p$ & $a c+c a r$ & $a c-1 c a r+$ & $a c-1 c a r$ \\
\hline & \multicolumn{7}{|c|}{ counts $/ \mathrm{min} . / \mathrm{mu} \times 10^{-4}$} \\
\hline $\begin{array}{l}\text { Respiratory } \\
\mathrm{CO}_{2}\end{array}$ & $2 \cdot 0$ & $2 \cdot 5$ & $\mathbf{2 \cdot 2}$ & $3 \cdot 7$ & $2 \cdot 5$ & $\mathbf{1 \cdot 9}$ & $3 \cdot 0$ \\
\hline Ethanol & $12 \cdot 7$ & $14 \cdot 6$ & $18 \cdot 0$ & $9 \cdot 7$ & $\mathbf{8 . 9}$ & $12 \cdot 7$ & $9 \cdot 7$ \\
\hline Ratio: & & & & & & & \\
\hline Ethanol $/ \mathrm{CO}_{2}$ & $6 \cdot 4$ & $5 \cdot 8$ & $8 \cdot 2$ & $2 \cdot 6$ & $3 \cdot 6$ & $6 \cdot 7$ & $3 \cdot 2$ \\
\hline
\end{tabular}

\section{Enzymic pattern}

To verify the results obtained in growth and isotope experiments, enzymic assays for ethanol dehydrogenase, aldolase and glucose-6-phosphate dehydrogenase were undertaken. Aldolase was studied because it is a key enzyme in the Embden-Meyerhof scheme, glucose-6-phosphate dehydrogenase because it is the first step in the pentose shunt, and ethanol dehydrogenase because of its obvious relation to accumulation and utilization.

Enzyme activity was measured after growth for 4 days on sucrose or xylose (Table 6). Ethanol-dehydrogenase assays were also made using cultures of $a c-3 s p$ and $a c-1$ car grown 3 days in the presence and absence of added ethanol (Table 7). It will be noted that the expected correlation between ethanol accumulation and ethanol dehydrogenase activity was obtained. Strains $a c s p$ and $a c-1$ car, which accumulate very little ethanol, have low ethanol dehydrogenase activities. When grown in the presence of ethanol, however, the dehydrogenase activity of these strains is increased, although it still does not approach the wild-type level (Table 7). This is in contrast to the effect of pyruvate on these strains. Both have low pyruvic carboxylase activities but growth with added pyruvate does not increase their carboxylase activities. All the mutant strains tested have lower ethanol dehydrogenase activities than the wild-type, in accordance with the findings of Nason, Kaplan \& Oldewurtal (1953).

Aldolase activity is given as $\mu \mathrm{M}$ of hexosediphosphate (HDP) split/hr./mg. 
protein N. HDP values were obtained as described by Sibley \& Lehninger (1949) by correlating the triose chromogen readings of their method with the alkali-labile P liberated. $N$. crassa does contain aldolase, and a linear correlation is obtained between enzyme concentration, alkali-labile $\mathbf{P}$ production and chromogen reading. The aldolase activities of the strains used in these investigations are similar with the exception of $a c-4 s p+$ which has low activity (Table 6) and there is no difference in activity after growth on xylose as compared to growth on sucrose. The lower wild-type aldolase activity may be due to the disappearance of sugar from the medium in which this strain is growing, after 3 days of growth.

\section{Table 6. Enzyme activities of ac strains}

Aldolase activity : $\mu \mathrm{M}-\mathrm{HDP}$ split/hr./mg. protein $\mathrm{N}$ at $37^{\circ}$. Glucose-6-phosphate dehydrogenase activity : increase in optical density at $340 \mathrm{~m} \mu$. $/ \mathrm{min}$./mg. protein N. Ethanol dehydrogenase activity: increase in optical density at $340 \mathrm{~m} \mu . / \mathrm{min} . / \mathrm{mg}$. protein $\mathrm{N}$.

\begin{tabular}{|c|c|c|c|}
\hline Strain & Aldolase & $\begin{array}{c}\text { Enzyme, } \\
\text { glucose-6- } \\
\text { phosphate } \\
\text { dehydrogenase }\end{array}$ & $\begin{array}{c}\text { Ethanol } \\
\text { dehydrogenas }\end{array}$ \\
\hline \multicolumn{4}{|c|}{ 1. Grown 4 days on $2 \%$ sucrose } \\
\hline$a c+c a r+s p+$ & $0 \cdot 44$ & 0.52 & $1 \cdot 43$ \\
\hline$a c+c a r$ & 0.55 & $0 \cdot 22$ & $0 \cdot 069$ \\
\hline$a c-1 c a r$ & $0 \cdot 80$ & $0 \cdot 20$ & 0.007 \\
\hline$a c+s p$ & $0 \cdot 65$ & 0.017 & $0 \cdot 043$ \\
\hline$a c-2 s p+$ & $0 \cdot 65$ & 0.11 & $0 \cdot 14$ \\
\hline$a c-3 s p+$ & 0.80 & 0.21 & $0 \cdot 12$ \\
\hline$a c-3 s p$ & $0 \cdot 75$ & $0 \cdot 13$ & $0 \cdot 004$ \\
\hline$a c-5 s p+$ & 0.75 & $0 \cdot 16$ & $0 \cdot 24$ \\
\hline$a c-5 s p$ & $0 \cdot 80$ & $0 \cdot 15$ & 0.0 \\
\hline \multicolumn{4}{|c|}{ 2. Grown 4 days on $1 \%$ xylose } \\
\hline$a c+c a r+s p+$ & 0.55 & $0 \cdot 22$ & $0 \cdot 20$ \\
\hline$a c-1 c a r+$ & $1 \cdot 0$ & 0.044 & 0.01 \\
\hline$a c-1 c a r$ & $0 \cdot 65$ & $0 \cdot 12$ & 0.009 \\
\hline$a c+s p$ & 0.55 & $0 \cdot 0$ & 0.001 \\
\hline$a c-4 s p+$ & $0 \cdot 13$ & 0.058 & 0.047 \\
\hline
\end{tabular}

\section{Table 7. Effect of ethanol on ethanol dehydrogenase activity}

Recorded activity/mg. protein $\mathrm{N} \times 10^{3}$ after 3 days growth at $25^{\circ}$ in 20 ml. minimal medium + acetate + addition as given.

$\begin{array}{lcc} & \overbrace{\text { Ntrain }} & \text { Ethanol } \\ a c+s p+ & \mathbf{5 7 0} & 490 \\ a c-3 s p & \mathbf{0} & \mathbf{5 8} \\ a c-5 s p & \mathbf{4} & \mathbf{6 6}\end{array}$

The $a c s p+$ and $a c c a r+$ strains used have at most $1 / 2$ to $1 / 3$ of the glucose6-phosphate dehydrogenase activity of wild-type. There is no difference in the activity of $a c s p$ and $a c s p+$ strains. The $a c+s p$ strain used in these investigations has an extremely low glucose-6-phosphate dehydrogenase activity, in marked contrast to the $a c+c a r$ strain which has an activity similar to the other mutants. Enzyme activity is lower after growth on xylose 
than after growth on sucrose, but the ac-1 car strain has three times the activity of ac-1 car + after growth on xylose. Since no genetic studies were made, these strain differences are not necessarily characteristic of the $s p$ and car genes.

\section{DISCUSSION}

The following hypothesis accounts for the data (Fig. 1). ac Mutants cannot oxidatively decarboxylate pyruvate to acetate (or active acetate). AMC accumulates. The pathway pyruvate $\rightarrow$ acetaldehyde $\rightarrow$ acetate is open and ac mutants make acetate via this route. In the early stages of growth $a c$ strains do not remove acetaldehyde rapidly enough. Acetaldehyde, or some substance formed from acetaldehyde, is inhibitory and is the cause of 'glucose inhibition'. After some time certain of the $a c$ strains acquire the ability to remove the formed acetaldehyde at a rate sufficient to keep its concentration below the inhibitory level. At that time, rapid growth ensues and continues, presumably on the acetate formed from acetaldehyde oxidations.

The introduction of either the $s p$ or $c a r$ gene into $a c$ strains results in relief from glucose inhibition, since a decrease of carboxylase activity induces a greatly lessened rate of acetaldehyde formation. Some acetaldehyde is still formed, however, and the oxidation of this to acetate leads to slow growth and the partial 'suppression' of the $a c$ mutants.

Lowering the rate of acetaldehyde formation lowers the ethanol dehydrogenase activity of $c a r$ and $s p$ strains by eliminating the substrate upon which this dehydrogenase acts. This is an example of the mechanism by which a single gene change can lead to a change in the activity of more than one enzyme by indirect means, since growth of $a c s p$ strains in the presence of ethanol partially restores enzyme activity (cf. Vogel \& Davis, 1952).

Prohibition of ethanol formation by $a c+$ strains increases the crop of mycelium obtained; $a c+s p$ strains have given nearly twice the dry weight of $a c+s p+$ strains under our conditions. There is a slight retardation of the initial growth rate caused by the $s p$ gene. Apparently the 'ethanol excursion' (Foster, 1949) is not a particularly efficient mechanism, but $a c+s p+$ strains have no method for controlling the amount of glucose they take up and must consequently accumulate ethanol.

This hypothesis is consistent with the growth data; the greater susceptibility of $a c s p+$ strains to ethanol and pyruvate inhibition is explained by the ability of these substances to serve as acetaldehyde precursors. The $a c$ strains may be more susceptible to acetaldehyde poisoning, rather than being poisoned by amounts of acetaldehyde greater than normal. Decision rests upon location of the precise biochemical lesions in pyruvate oxidation in the different non-allelic acetate mutants. While some compound derived from acetaldehyde may be the actual inhibitor, because of the relatively high concentrations required for inhibition, the relationship to acetaldehyde must be close, since the non-allelic genes $s p$ and $c a r$ both relieve glucose inhibition and are both associated with low carboxylase activity and since no inhibition is noticed when growth occurs on xylose, which similarly leads to low carboxylase activity. 
Altered pathways of metabolism. Although ac $s p$ mutants do form more of their initial respiratory $\mathrm{CO}_{2}$ from the 1-carbon of glucose than do ac $s p+$ strains, there is no evidence that this is a direct result of the $s p$ mutation. Apparently the introduction of a double block in the pyruvic acid metabolism slows down glucose metabolism and retards the steady-state operation of the Embden-Meyerhof path. Since glucose can no longer be metabolized easily by this route, an alternate pathway is used to a relatively greater extent, giving the 1-carbon of glucose as $\mathrm{CO}_{2}$. If this alternate path utilizes the action of glucose-6-phosphate dehydrogenase, then ac sp strains are not able to increase the amount of enzyme sufficiently to take care of glucose at the total wild-type rate. Pyruvate is accumulated by $a c s p$ strains to a greater extent than by ac sp+, but if the above interpretation is correct, the pyruvate accumulated by $a c s p$ has a different metabolic history than the lesser amounts of pyruvate accumulated by the $a c s p+$ strain. The car gene, however, seems to act by shunting metabolism along an alternate route in accordance with the original hypothesis, since the $a c+c a r$ strain tested has a lower ethanol $/ \mathrm{CO}_{2}$ activity ratio than the wild-type. If these interpretations are correct, then $c a r$ and $s p$, which are genetically non-allelic, lower carboxylase activity by different mechanisms.

Mechanism of suppressor action. The mechanism for suppressor action derived from these studies is very similar to that proposed by Emerson (1948) for suppression of the sfo characteristic. It was supposed by Emerson and later by Horowitz (1951) that this mechanism was not of general applicability. However, it would seem that this type of suppressor action might well be more common than is supposed, especially since so many single gene mutations seem to result in inhibition by normal metabolites. Suppressors may often be 'loss' mutations which prevent the formation or accumulation of inhibitory substances formed as a result of a primary mutation. Since such suppressed mutants have essentially two biochemical lesions it might be expected that they would approach but not reach the true wild-type phenotype as is the case for the ac sp mutants. Giles (1951) and Giles \& Partridge (1953) described certain suppressors of the methionineless and inositolless characteristics that similarly approach but do not reach the wild-type phenotype. Similarly, Mitchell \& Mitchell (1952) described a whole series of inhibitory phenomena connected with the action of a suppressor gene.

The authors are indebted to Miss M. L. Jezyk and to J. E. Matheson for technical assistance, and to Dr S. H. Hutner for helpful criticism of the manuscript.

The work was supported by contract AT (30-1)-1138 between Syracuse University and the (U.S.) Atomic Energy Commission.

\section{REFERENCES}

Ballentine, R. \& GregG, J. R. (1947). Micro-Kjeldahl determination of nitrogen. Analyt. Chem. 19, 281.

Barker, J. B. \& Summerson, W. H. (1941). The colorimetric determination of lactic acid in biological material. J. biol. Chem. 138, 535.

Beadle, G. W. (1945). Biochemical genetics. Chem. Rev. 37, 15. 
Beadle, G. W. \& Tatum, E. L. (1945). Neurospora. II. Methods of producing and detecting mutations concerned with nutritional requirements. Amer. J. Bot. $32,678$.

Bonner, D. (1946). Further studies of mutant strains of Neurospora requiring isoleucine and valine. J. biol. Chem. 166, 545.

Dixon, M. (1949). Multi-Enzyme Systems. Cambridge University Press.

Emerson, S. (1948). A physiological basis for some suppressor mutations and possibly for one gene heterosis. Proc. nat. Acad. Sci., Wash. 34, 72.

Emerson, S. (1949). Competitive reactions and antagonisms in the biosynthesis of amino acids by Neurospora. Cold Spr. Harb. Symp. quant. Biol. 14, 40.

Fiske, C. H. \& SubbaRow, Y. (1925). The colorimetric determination of phosphorus. J. biol. Chem. 66, 375.

Friedemann, T. E. \& Klass, R. (1936). The determination of ethyl alcohol. J. biol. Chem. 115, 47.

Friedemann, T. E. \& Haugen, G. E. (1943). Determination of keto acids in blood and urine. J. biol. Chem. 147, 415.

Foster, J. W. (1949). Chemical Activities of Fungi. New York: Academic Press Inc.

Giles, N. H. (1951). Studies on the mechanism of reversion in biochemical mutants of Neurospora crassa. Cold Spr. Harb. Symp. quant. Biol. 16, 283.

Giles, N. H. \& Partridge, C. W. H. (1953). The effect of a suppressor on allelic inositolless mutants in Neurospora crassa. Proc. nat. Acad. Sci., Wash. 39, 479.

Horowitz, N. H. (1950). Biochemical genetics of Neurospora. Advanc. Genet. 3, 33.

Horowitz, N. H. (1951). Genetic and non-genetic factors in the production of enzymes by Neurospora. Growth, Symp. 10, 47.

Lein, J., Appleby, D. C. \& Lein, P. S. (1951). Acetate formation in Neurospora studied with biochemical mutants. Arch. Biochem. Biophys. 34, 72.

LEIN, J. \& Lein, P. S. (1952). Studies on a suppressor of non-allelic acetate requiring mutants of Neurospora. Proc. nat. Acad. Sci., Wash. 38, 44.

Mitchell, M. B. \& Mitchell, H. K. (1952). Observations on the behaviour of suppressors in Neurospora. Proc. nat. Acad. Sci., Wash. 38, 205.

Nason, A., Kaplan, N. O. \& Colowick, S. P. (1951). Changes in enzymatic constitution in zinc-deficient Neurospora. J. biol. Chem. 188, 397.

Nason, A., Kaplan, N. O. \& Oldewurtal, H. A. (1953). Further studies of nutritional conditions affecting enzymatic constitution in Neurospora. J. biol. Chem. 201, 435.

Ponteconvo, G. (1952). Genetic formulation of gene structure and gene action. Advanc. Enzymol. 13, 121.

Shaffer, P. A. \& Somogyi, M. J. (1933). Copper-iodometric reagents for sugar determination. J. biol. Chem. 100, 695 .

Sibley, J. A. \& Lehninger, A. L. (1949). Determination of aldolase in animal tissues. J. biol. Chem. 177, 859.

STrauss, B. S. (1952). Aspects of the carbohydrate metabolism of a mutant of Neurospora crassa requiring acetate for growth. Arch. Biochem. Biophys. 36, 33.

Strauss, B. S. (1953a). The accumulation of acetylmethylcarbinol (3-hydroxy-2butanone) by acetate-requiring mutants of Neurospora crassa. J. Amer. chem. Soc. 75, 1012.

Strauss, B. S. (1953b). Properties of mutants of Neurospora crassa with low pyruvic carboxylase activity. Arch. Biochem. Biophys. 44, 200.

Vogel, H. J. \& Davis, B. D. (1952). Adaptive phenomena in a biosynthetic pathway. Fed. Proc. 11, 485.

Westerfeld, W. W. (1945). A colorimetric determination of blood acetoin. J. biol. Chem. 161, 495. 